\title{
Effect of neutrophil depletion on gelatinase expression, edema formation and hemorrhagic transformation after focal ischemic stroke
}

\author{
Alex K Harris ${ }^{1}$, Adviye Ergul1,2, Anna Kozak1, Livia S Machado1, \\ Maribeth H Johnson ${ }^{4}$ and Susan C Fagan*1,3,5
}

\begin{abstract}
Address: ${ }^{1}$ Program in Clinical and Experimental Therapeutics, College of Pharmacy, University of Georgia, Augusta, Georgia, USA, ${ }^{2}$ Vascular Biology Center, Medical College of Georgia, Augusta, Georgia, USA, ${ }^{2}$ Veteran's Affairs Medical Center, Medical College of Georgia, Augusta, Georgia, USA, ${ }^{4}$ Department of Biostatistics, Medical College of Georgia, Augusta, Georgia, USA and ${ }^{5}$ Department of Neurology, Medical College of Georgia, Augusta, Georgia, USA

Email: Alex K Harris - aharris@mail.mcg.edu; Adviye Ergul - aergul@mail.mcg.edu; Anna Kozak - akozak@mail.mcg.edu;

Livia S Machado - Imachadogs@mail.mcg.edu; Maribeth H Johnson - majohnso@mail.mcg.edu; Susan C Fagan* - sfagan@mail.mcg.edu

* Corresponding author
\end{abstract}

Published: 03 August 2005

BMC Neuroscience 2005, 6:49 doi:10.1 I86/147|-2202-6-49

This article is available from: http://www.biomedcentral.com/I47I-2202/6/49

(C) 2005 Harris et al; licensee BioMed Central Ltd.

This is an Open Access article distributed under the terms of the Creative Commons Attribution License (http://creativecommons.org/licenses/by/2.0), which permits unrestricted use, distribution, and reproduction in any medium, provided the original work is properly cited.
Received: 3I March 2005

Accepted: 03 August 2005

\begin{abstract}
Background: While gelatinase (MMP-2 and -9) activity is increased after focal ischemia/ reperfusion injury in the brain, the relative contribution of neutrophils to the MMP activity and to the development of hemorrhagic transformation remains unknown.

Results: Anti-PMN treatment caused successful depletion of neutrophils in treated animals. There was no difference in either infarct volume or hemorrhage between control and PMN depleted animals. While there were significant increases in gelatinase (MMP-2 and MMP-9) expression and activity and edema formation associated with ischemia, neutrophil depletion failed to cause any change.

Conclusion: The main finding of this study is that, in the absence of circulating neutrophils, MMP2 and MMP-9 expression and activity are still up-regulated following focal cerebral ischemia. Additionally, neutrophil depletion had no influence on indicators of ischemic brain damage including edema, hemorrhage, and infarct size. These findings indicate that, at least acutely, neutrophils are not a significant contributor of gelatinase activity associated with acute neurovascular damage after stroke.
\end{abstract}

\section{Background}

The matrix metalloproteinases (MMPs) are a family of some 23 zinc dependent proteases that, collectively, possess the ability to degrade nearly every component of the extra-cellular matrix [1-3]. The activity of the MMPs is tightly controlled through proteolytic activation of the zymogen forms and stoichiometric binding of tissue inhibitors of metalloproteinases (TIMPs). The MMPs play an important role in many physiological processes due to their inherent ability to remodel tissues [2,3]. However, in disease states such as vascular disease and stroke, the MMPs may become deleterious due to dysregulation and can result in tissue injury and inflammation. Specifically, the MMPs may be involved in the degradation of the basal 
lamina in reperfusion injury resulting in disruption of the blood brain barrier and hemorrhagic transformation [4].

Recently, several lines of evidence have demonstrated the involvement of the MMPs in cerebral ischemia. Studies in rat, mouse, and baboon models have shown that MMP-9 is up-regulated following transient focal ischemia [5-8]. Additionally, Asahi et al. have shown that MMP-9 knockout as well as MMP-9 inhibition reduces ischemic lesion volume [9]. However, others have shown that pharmacological inhibition of MMP-9 yields no change $[10,11]$. Lapchak et al. demonstrated that broad-spectrum MMP inhibition (BB-94) reduced the incidence of hemorrhage in tPA treated brains when administered shortly after the onset of ischemia [11]. In addition, Sumii et al. were able to show a reduction in hemorrhage severity in tPA treated animals given the same MMP inhibitor BB-94 [10].

Clearly the MMPs are involved in the pathology of cerebral ischemia and hemorrhagic transformation. However, there is still uncertainty as to the origin of MMP activity. Immunohistochemical studies from Asahi et al. demonstrated that MMP-9 is primarily up-regulated in the vascular spaces while others have shown that there is a concomitant staining of neutrophils in the areas of MMP9 activation suggesting a role for the neutrophil in the upregulation of MMP-9 [12-14]. Indeed, a recent study has shown that prevention of neutrophil infiltration significantly reduces MMP-9 up-regulation in an occlusion/ reperfusion model of ischemia [15]. The source of MMP activity in focal cerebral ischemia is important to the development of therapies to target this mediator of neurovascular injury. It is still unknown whether neutrophils are an important source of MMPs in experimental hemorrhagic transformation.

The objective of the current study was to evaluate the role of the neutrophil in hemorrhagic transformation and edema formation in a hyperacute 3-hour occlusion/reperfusion model of focal ischemia. The central hypothesis was that depletion of neutrophils would reduce hemorrhage development due to prevention of the up-regulation of MMP-9.

\section{Results}

\section{Neutrophil depletion}

Control and neutrophil-depletion groups received normal rabbit serum and anti-PMN antibody, respectively, 24 hours prior to middle cerebral artery occlusion (MCAO) surgery. The dose of the anti-neutrophil antibody chosen (and this was batch-specific in our preliminary studies not shown) was very effective in reducing the circulating neutrophils. In control animals that received normal serum, neutrophil count, expressed as mean \pm SE of percent of total leukocyte count, was $11.8 \pm 0.92 \%$ and as

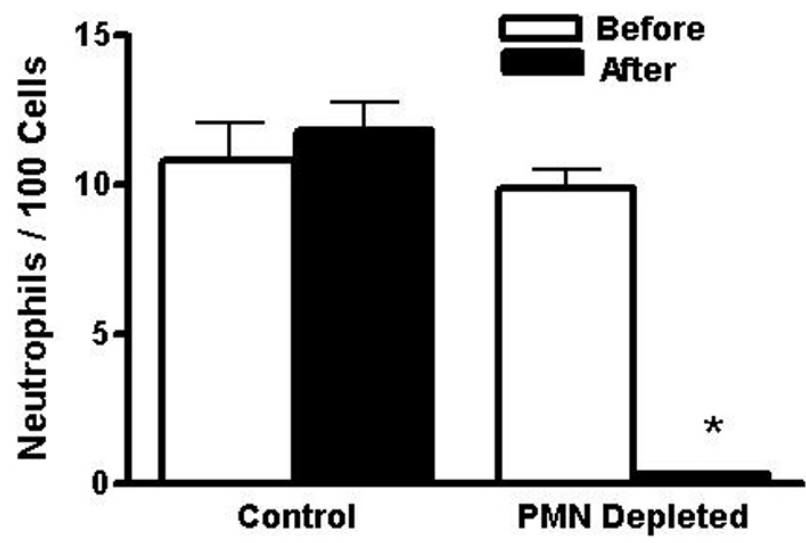

\section{Figure I}

Neutrophil counts at baseline and at 24 hours after normal rabbit serum (control) or anti-PMN antibody (PMNdepleted) administration. Anti-PMN antibody resulted in a consistent decrease in circulating neutrophils to less than $10 \%$ of baseline counts whereas there was no change in neutrophil counts in control animals that received the normal rabbit serum. ${ }^{*} p<0.001$ control $(n=8)$ vs after PMN-depletion $(n=10)$.

expected did not differ from baseline. In the depletion group, administration of anti-PMN antibody reduced the neutrophil count to $0.3 \pm 0.11 \%$ from $9.4 \%$ at baseline ( $p$ $<0.0001$ compared to serum). All animals included in the neutropenic group had $>90 \%$ depletion of their neutrophils prior to the stroke surgery, as assessed by a blinded investigator using a hemocytometer (Fig. 1). The body weight of control animals was $288 \pm 4 \mathrm{~g}$ at baseline, $292 \pm 4 \mathrm{~g}$ prior to stroke and $237 \pm 4 \mathrm{~g}$ at 24 hours. In the antibody-treated group, body weight was $300 \pm 4 \mathrm{~g}$ at baseline, $292 \pm 5$ prior to stroke surgery, and $240 \pm 5 \mathrm{~g}$ at 24 hours.

\section{Gelatinase quantification}

Ischemia for three hours followed by $24 \mathrm{~h}$ reperfusion resulted in a significant increase in the expression and activity of both MMP-2 and MMP-9 in the ipsilateral hemispheres (Fig. 2). Quantitative analysis of the gelatin zymography revealed consistent increases in the lower molecular weight form of MMP-9 (88 kDa). Although there was a faint band corresponding to $95 \mathrm{kDa} M M P$, the gelatinolytic activity in control vs neutrophil-depleted animals was similar. Protein levels of MMP-2 (Fig. 3) and MMP-9 (Fig. 4) were significantly increased in the ischemic hemisphere and neutrophil depletion did not affect MMP protein levels. 
PMN-depleted

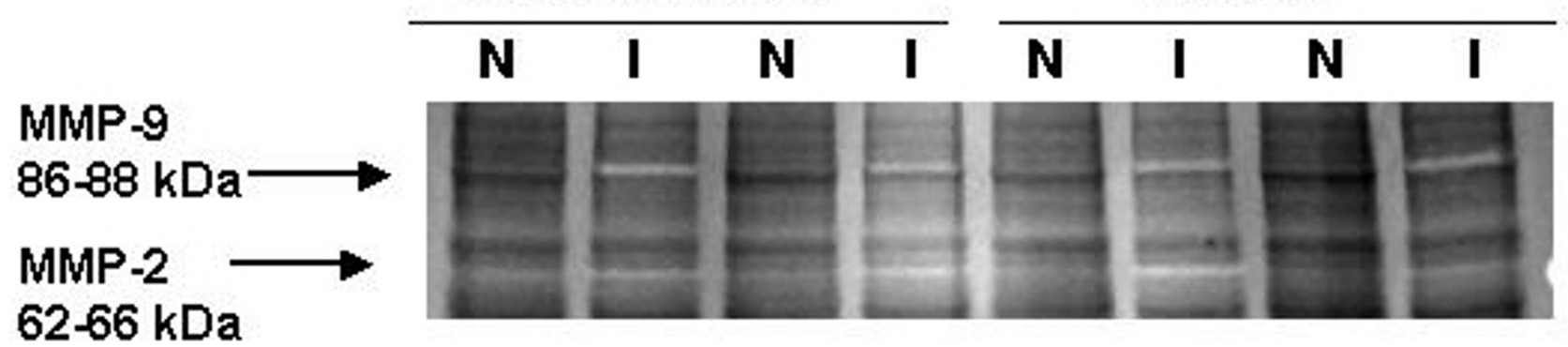

MMP-2

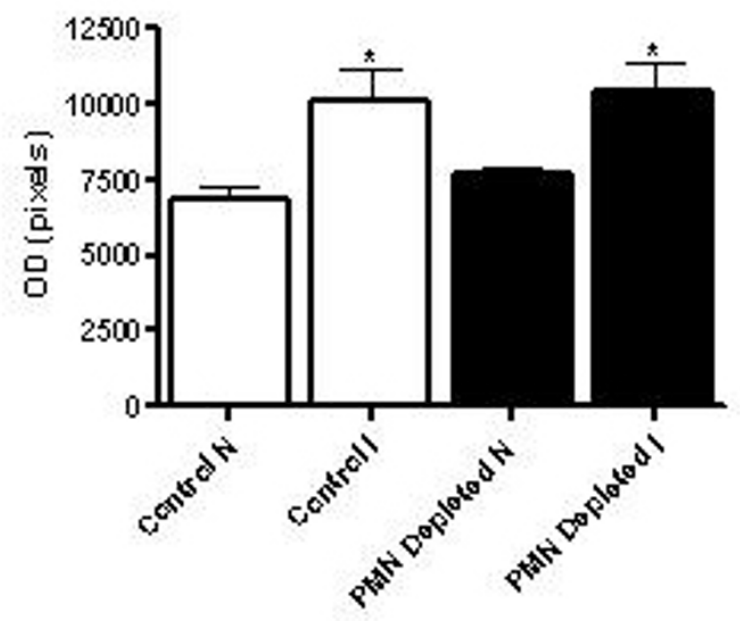

MMP-9

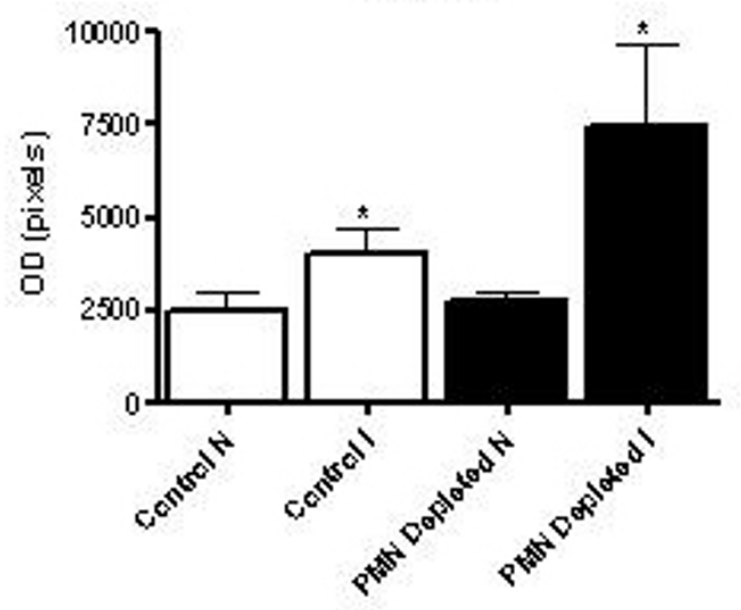

Figure 2

MMP activity is increased in focal cerebral ischemia. (A) Representative zymogram showing changes in MMP activity in the ischemic (I) and non-ischemic (N) hemispheres. (B) Densitometric analysis of lytic bands indicates increases in MMP-2 and MMP-9 activity in ischemic hemispheres from control and PMN-depleted animals. Anti-PMN antibody treatment has no affect on MMP activity. ( $* 0.05$ vs contralateral hemisphere, control $n=6, P M N n=4)$. 
PMN-depleted

\section{$\mathbf{N}$}

$\mathbf{N}$

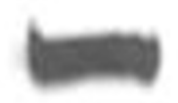

\section{$\mathbf{N}$}

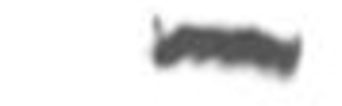

\section{Control}

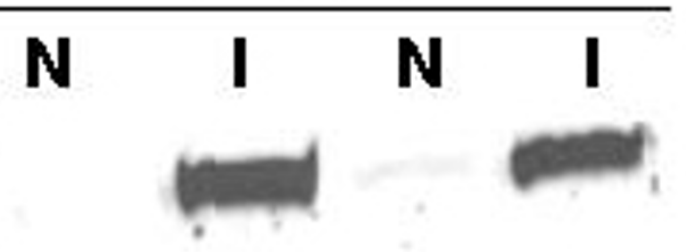

\section{MMP-2}

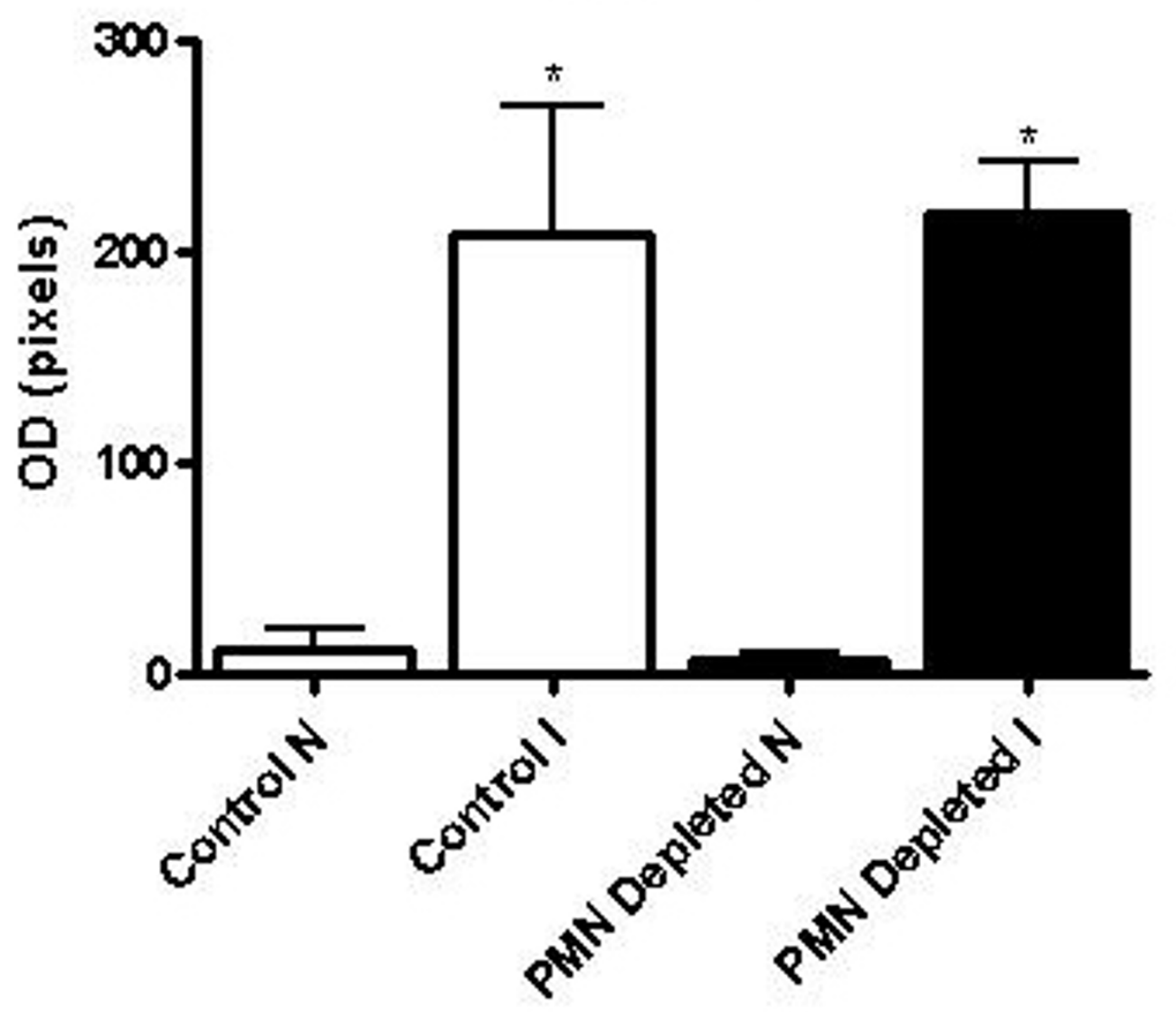

Figure 3

MMP-2 protein expression is increased in ischemia. (A). Representative immunoblot demonstrating changes in MMP-2 protein expression in ischemic hemispheres from control and PMN-depleted animals. (B) Densitometric analysis of immunoreactive bands indicates that MMP-2 protein is increased in ischemia and is unchanged by neutrophil depletion. ( ${ }^{*} \mathrm{p}<0.05$ vs contralateral hemisphere, saline $n=6, P M N n=4$ ). 
PMN-depleted

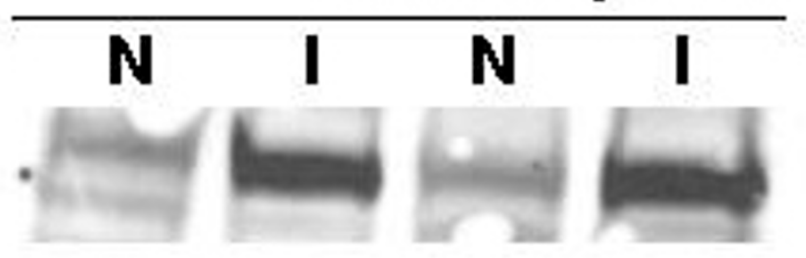

\section{Control}

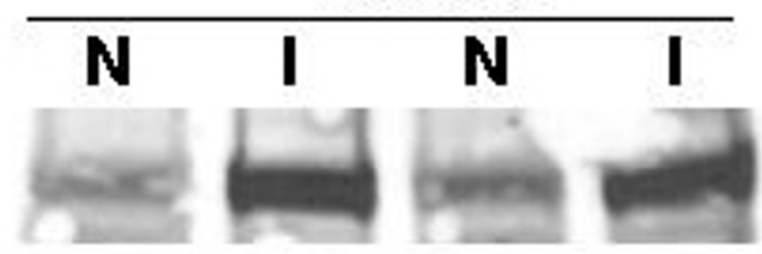

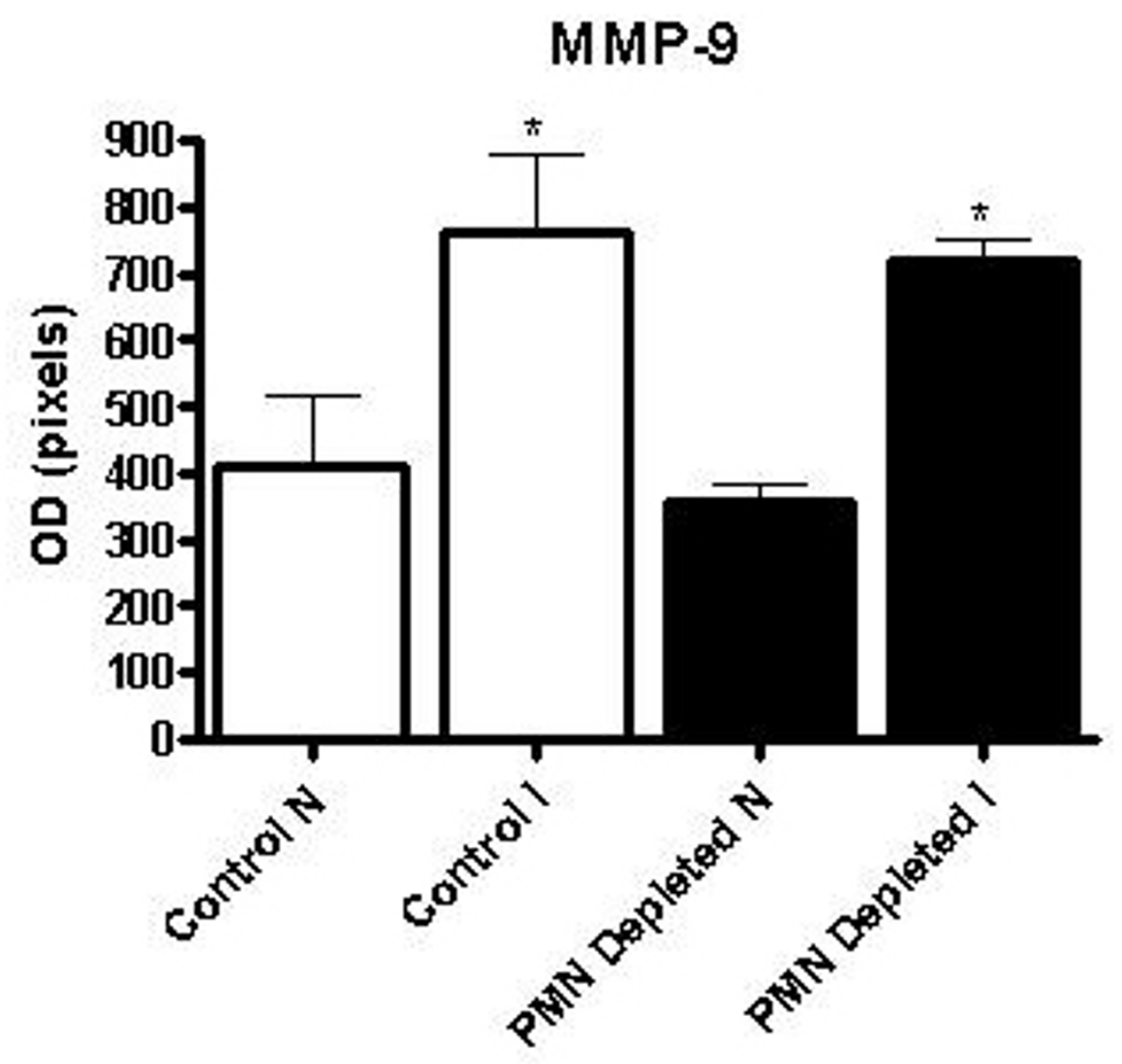

Figure 4

MMP-9 protein expression is unchanged by neutrophil depletion. (A). Representative immunoblot showing immunoreactive MMP-9 bands in both ischemic (I) and non-ischemic $(N)$ hemispheres. (B) Densitometric analysis indicates an ischemia induced elevation of MMP-9 expression that is unaffected by PMN depletion. ( $*^{*}<0.05$ vs contralateral hemisphere, control $n=6$, PMN n = 4). 


\section{Infarct size, edema and hemorrhage assessment}

There was no significant effect of neutrophil depletion on either infarct size or the development of hemorrhagic transformation in our model (Figure 5). In addition, cerebral edema formation was similar between untreated and neutrophil-depleted animals. Neurologic examination at 24 hours was not significantly different between the two treatment groups (not shown).

\section{Discussion}

Cerebral ischemia and reperfusion are known to induce large increases in MMP-9 protein and activity in the affected hemisphere and MMP-9 has been associated with hemorrhage formation in humans [16] and experimental animals [10]. Experimental inhibition of the MMPs has been associated with decreased hemorrhage formation and improved stroke outcome [11]. Since neutrophils are a known source of MMP-9, this study was designed to determine whether neutrophil depletion, prior to cerebral artery occlusion, would decrease MMP expression and reduce hemorrhagic transformation.

Despite the fact that neutrophil depletion was essentially complete prior to the focal cerebral ischemia in our experiment, we detected an increase in gelatinolytic activity corresponding to 86-88 kDa MMP-9. There was a faint band for the $95 \mathrm{kDa}$ proMMP-9, and the activity did not differ between the ischemic and non-ischemic hemispheres. Immunoblotting experiments confirmed the results of gelatin zymography and showed that specific up-regulation of the MMP-9 protein is responsible for increased gelatinolytic activity. Justicia et al. reported that, in a $1 \mathrm{~h}$ MCAO model in which the neutrophil depletion or antagonism was achieved by vinblastine administration or neutralizing antibodies against VCAM-1, respectively, there was no effect on the $88 \mathrm{kDa}$ MMP-9 expression but, increases in $95 \mathrm{kDa}$ MMP-9 activity were prevented. Our results are in agreement with this observation that neutrophils are not the source of 88 kDa MMP-9. However, we do not detect any increase in 95 kDa MMP-9. This difference may be due to the duration of ischemia ( $1 \mathrm{~h}$ vs 3 h) and the extent of ischemic damage [15]. We have previously shown that hemorrhagic transformation occurs in various different regions of the injured hemisphere including: preoptic area, striatum and the lateral cortex in this model and that development of hemorrhagic transformation is related to the duration of occlusion, with no hemorrhage when reperfusion occurs after only 1 hour $[17,18]$.

In this model of focal cerebral ischemia and hemorrhagic transformation, where the MCA was occluded for 3 hours prior to reperfusion, no significant effect of neutrophil depletion could be shown on either neurovascular damage or neurologic function. Also, from our results, it is clear that neutrophils are not an important contribution to the increased MMP-9 expression in the 24 hours after this injury. It is possible that the contributions of neutrophils are greater when the injury to the brain is less profound than in our model. Several different investigators have demonstrated that preventing the adhesion of neutrophils [19] after ischemia and reperfusion reduces the ultimate injury. Maier et al. reported that in superoxide dismutase (SOD) knock-out animals that are more susceptible to ischemic damage, neutrophils are not the source of MMP-9 contributing to blood brain barrier breakdown providing further support for the results of this present study [20].

Activated neutrophils release free radicals and proteolytic enzymes such as MMP-8 (neutrophil elastase), in addition to activating cytokines, which further the recruitment of leukocytes to the site of injury. We initially thought that neutrophil adherence and activation may be an early contributor to the development of microvascular injury and hemorrhagic transformation after cerebral ischemia but this turned out to be not the case [21]. It is still possible that neutrophils are involved in the destruction of the basal lamina, but it is likely to occur later in the process, after neutrophil recruitment is maximal.

\section{Conclusion}

Although neutrophils have been shown to contain MMP9, release of MMP-9 by neutrophils is probably not the mechanism of the early microvascular damage leading to edema formation and hemorrhagic transformation in our model. In addition, neutrophil adhesion is not necessary for the increase in activation of MMP-9 after ischemia. Although neutrophils may contribute to the ultimate degree of neurologic damage after ischemia and reperfusion in the brain, they are not necessary for the development of hemorrhagic transformation.

\section{Methods}

\section{Neutrophil depletion}

Neutrophil depletion was accomplished by intravenous administration of a polyclonal rabbit anti-rat polymorphonuclear neutrophil (PMN) antiserum (Accurate Chemical and Scientific Corporation, Westbury, NY) 24 hours prior to ischemia [22]. Animals were randomized to receive either the PMN antiserum $(0.3 \mathrm{~mL}$ diluted in $1 \mathrm{~mL}$ saline) or an equal volume of normal rabbit serum (control) 24 hours prior to surgery. Neutrophil counts, as a percentage of total leukocyte count, were determined at baseline and 23 hours post administration to determine the level of depletion.

\section{Animal preparation/physiological monitoring}

The Care of Experimental Animal Committee of Medical College of Georgia approved the protocol. Male Wistar 


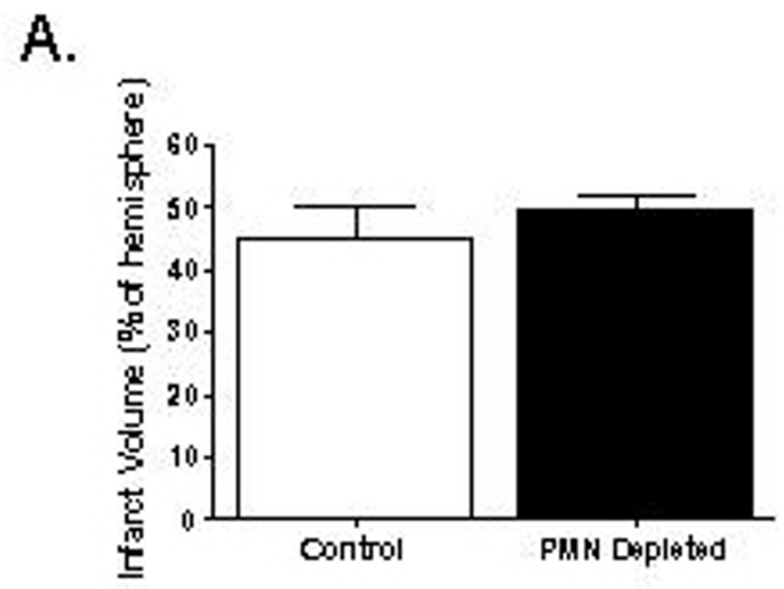

B.
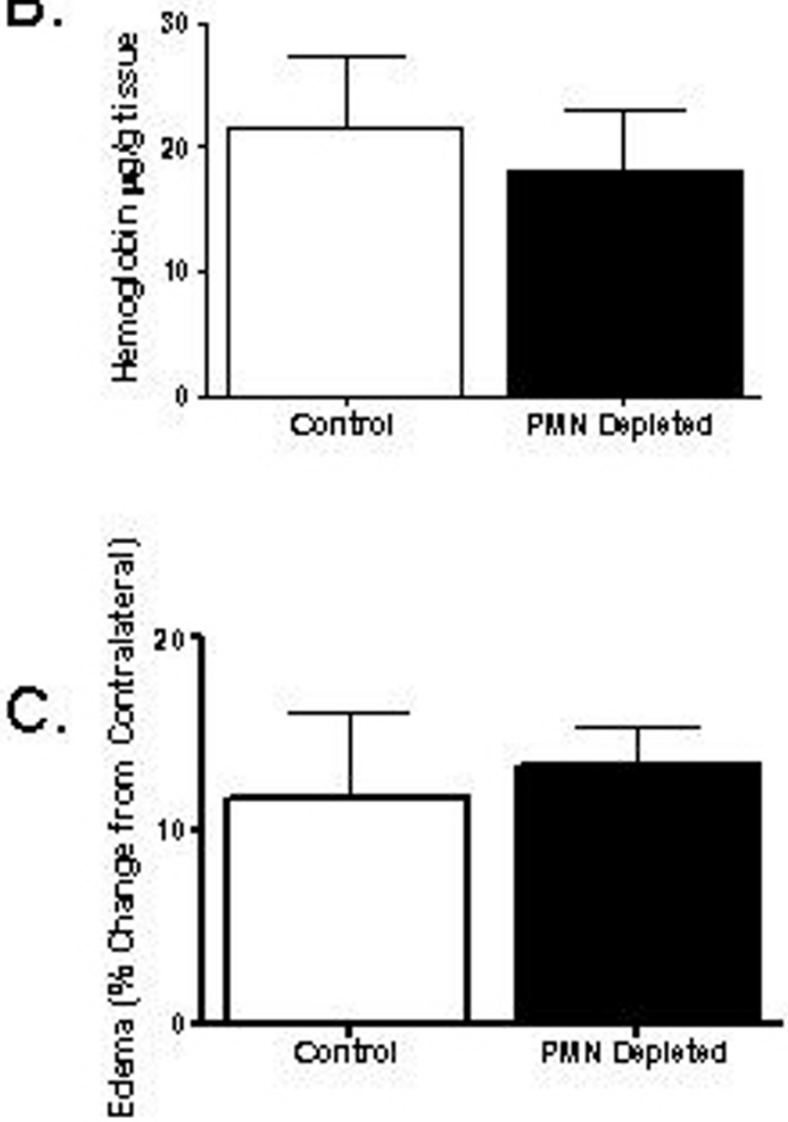

Figure 5

Infarct size, edema formation and hemoglobin excess differences between control and PMN-depleted animals. (A). Infarct size, as measured by TTC staining, indicated no differences between depletion and control groups. (B). Likewise, PMN depletion had no affect on the development of hemorrhagic transformation. (C) Edema determined as the difference in area between the ischemic and non-ischemic hemispheres was similar in neutrophil-depleted and control animals $(n=8$ control, $n=10$ PMN). 
rats, from the Charles River Breeding Company (Wilmington, MA, USA) within a narrow range of body weight (250-300 g) were used. All animals were anesthetized with $2 \%$ isoflurane via inhalation. Cerebral ischemia was induced using the intraluminal suture MCAO model [23]. The MCA was occluded with a 19-21 mm 4-0 surgical nylon filament, which was introduced from the external carotid artery lumen into the internal carotid artery to block the origin of the MCA. The suture was removed after 3 hours of occlusion. We have shown previously that hemorrhagic transformation is related to the duration of occlusion in this model $[17,18]$. Prior to, and after reperfusion, each rat was evaluated neurologically using the Bederson Scale [24]. After 21 hours of reperfusion, the animals were anesthetized with ketamine $44 \mathrm{mg} / \mathrm{kg}$ and xylazine $13 \mathrm{mg} / \mathrm{kg}$ I.M. (cocktail), perfused with saline, sacrificed, and the brain tissue was removed.

\section{Brain preparation and homogenization}

The brains were sliced into seven $2-\mathrm{mm}$ thick slices in the coronal plane and stained with a $2 \%$ solution of $2,3,5$ triphenyltetrazolium chloride (TTC) (Sigma Chemical Co., USA) for 15-20 minutes. Images of the stained sections were taken. Grossly visible infarction zones were quantified using image analysis software (Zeiss- KS 300) in pixel $^{2}$ [25]. Because brain edema can affect the accuracy of infarct assessment [26], the measurement of the corrected infarct size was taken $[27,28]$. The ischemic and non-ischemic hemispheres of the slices for the ELISA assay were separated and processed individually, using the non-ischemic side as a control. Each slice was homogenized, vortexed and sonicated. Quantification of the hemoglobin was accomplished using a direct ELISA method, as previously described [29].

For zymography and western blotting, brains were placed in a chilled coronal matrix and 2-mm sections were removed from the core of the infarct. The slices were collectively placed, by hemisphere, into labeled tubes, flash frozen, and stored at $-80^{\circ} \mathrm{C}$ until use. Samples were homogenized according to the methods of Heo et al. [7]. Briefly, samples were placed in $300 \mu \mathrm{L}$ cold working buffer consisting of $50 \mathrm{mmol} / \mathrm{L}$ Tris-HCl (pH 7.5), 75 $\mathrm{mmol} / \mathrm{L} \mathrm{NaCl}$, and $1 \mathrm{mmol} / \mathrm{L}$ PMSF, homogenized with a glass homogenizer and centrifuged at $4^{\circ} \mathrm{C}$ for 20 minutes at $9000 \mathrm{rpm}$. The supernatant was collected and stored at $-80^{\circ} \mathrm{C}$ until use. Protein concentrations were determined using the Bradford method.

\section{Gelatin zymography}

On the day of the experiment, samples $(20 \mu \mathrm{g}$ protein/ sample) were loaded onto $10 \%$ gelatin zymogram gels (Bio-Rad) and separated under nonreducing conditions [30]. The gels were then rinsed twice in 2.5\% Triton X-100 and incubated for 24 hours in substrate buffer containing
$21 \mathrm{mM}$ Tris $\cdot \mathrm{HCl}, 10 \mathrm{mM} \mathrm{CaCl}{ }_{2}, 0.04 \% \mathrm{NaN}_{3}$. Gels were then stained by Coomassie blue R-250 followed by destaining in 55\% methanol and 7\% acetic acid. Lytic activity was viewed as clear bands on a dark blue background and was quantitated by densitometric analysis (Gel-Pro v 3.1, Media Cybernetics, Carlsbad, CA.).

\section{Edema quantitation}

Brain edema was measured as a difference in area between the ischemic and non-ischemic hemispheres. The brain section analyzed was the one corresponding to the area of the ischemic damage in four consecutive $2-\mathrm{mm}$ slides. The brain slides were analyzed separately and the areas and differences combined for whole hemi-section comparison. The area analysis was done using Zeiss KS-300.

\section{Statistics}

A Wilcoxon rank sum test was performed to make group comparisons (control vs. depletion) in MMP activity and protein expression for the difference between the ischemic and non-ischemic sides of the brain and group comparisons for neutrophil depletion, edema, infarct size and hemorrhagic transformation. A Wilcoxon signed rank test was used to make within rat comparisons between ischemic and non-ischemic sides of the brain for MMP activity and protein expression. Statistical significance was determined at $\mathrm{p}<0.05$ and SAS 8.2 was used for all analyses.

\section{Authors' contributions}

AKH performed all the zymography and western blotting and contributed to preparation of the manuscript.

AE contributed to the optimization of zymograms, data interpretation and preparation of the manuscript.

AK did all the animal preparation, MCAO, infarct and hemoglobin analysis.

LM completed the edema analysis and contributed to the manuscript preparation.

MHJ performed the statistical analysis.

SCF coordinated all aspects of this work and prepared the manuscript.

All authors approved the final version of the manuscript.

\section{Acknowledgements}

This work was supported by grants from NINDS (IU0 INS43 I 27-0I and ROINS0442 16-0I) and the American Heart Association Southeast Affiliate to SCF, American Diabetes Association Research Award and NIH (HL076236-0I) awards to AE and American Heart Association Southeast Affiliate predoctoral fellowship to $\mathrm{AKH}$. 


\section{References}

I. Nagase H, Woessner JF: Matrix metalloproteinases. J Biol Chem I999, 274(3I):2|49|-2|494.

2. Visse $\mathrm{R}$, Nagase $\mathrm{H}$ : Matrix metalloproteinases and tissue inhibitors of metalloproteinases: structure, function, and biochemistry. Circ Res 2003, 92(8):827-839.

3. Smeglin A, Frishman WH: Elastinolytic matrix metalloproteinases and their inhibitors as therapeutic targets in atherosclerotic plaque instability. Cardiol Rev 2004, I2(3): | 4 | - I 50.

4. Hammann GF, Okada Y, Fritge R, del Zoppo G]: Microvascular basal lamina antigens disappear during cerebral ischemia and reperfusion. Stroke 1995:2 I20-2126.

5. Rosenberg GA, Navratil M, Barone F, Feuerstein G: Proteolytic cascade enzymes increase in focal cerebral ischemia in rat. Cereb Blood Flow Metab 1996, I6(3):360-366.

6. Gasche Y, Fujimura Y, Copin J, Kawase M, Masengale J, Chan PH: Early appearance of activated MMP-9 after focal cerebral ischemia in mice. J Cereb Blood Flow Metab 1999, I9:1020-1028.

7. Heo JH, Lucero J, Abumiya T, Koizol JA, Copeland BR, del Zoppo G]: Matrix metalloproteinases increase very early during experimental focal cerebral ischemia. J Cereb Blood Flow Metab 1999, 19:624-633.

8. Mun-Bryce S, Rosenberg GA: Matrix metalloproteinases in eerebrovascular disease. J Cereb Blood Flow Metab 1998, I 8: I I 63-I I 72

9. Asahi M, Asahi K, Jung J, del Zoppo GJ, Fini E, Lo EH: Role for matrix metalloproteinase 9 after focal cerebral ischemia: Effects of gene knockout and enzyme inhibition with BB-94. J Cereb Blood Flow Metab 2000, 20: I68I-1689.

10. Sumii T, Lo EH: Involvement of matrix metalloproteinase in thrombolysis-associated hemorrhagic transformation after embolic focal ischemia in rats. Stroke 2002, 33:83 I-836.

II. Lapchak PA, Chapman DF, Zivin JA: Metalloproteinase inhibition reduces thrombolytic (tissue plasminogen activator)induced hemorrhage after thromboembolic stroke. Stroke 2000, 3 I ( I 2):3034-3040.

12. Asahi M, Wang X, Mori T, Sumii T, Jung J, Moskowitz MA, Fini E, Lo $\mathrm{EH}$ : Effects of matrix metalloproteinase-9 gene knock-out on the proteolysis of blood-brain barrier and white matter components after cerebral ischemia. J Neurosci 200I, 2 I :7724-7732.

13. Planas AM, Justicia C, Sole S, Friguls B, Cervera A, Adell A, Chamorro $A$ : Certain forms of matrix metalloproteinase-9 accumulate in the extracellular space after microdialysis probe implantation and middle cerebral artery occlusion/reperfusion. J Cereb Blood Flow Metab 2002, 22:918-925.

14. Romanic AM, White RF, Arleth AJ, Ohlstein EH, Barone F: Matrix metalloproteinase expression increases after cerebral focal ischemia in rats: inhabition of matrix metalloproteinase- 9 reduces infarct size. Stroke 1998, 29:1020-1030.

15. Justicia C, Panes J, Sole S, Cervera A, Deulofeu R, Chamorro A, Planas AM: Neutrophil infiltration increases matrix metalloproteinase-9 in the ischemic brain after occlusion/reperfusion of the middle cerebral artery in rats. J Cereb Blood Flow Metab 2003 23:|430-|440.

16. Montaner J, Alvarez-Sabin J, Molina C, Angles A, Abilleira S, Arenillas J, Monasterio J: Matrix metalloproteinase expression is related to hemorrhagic transformation after cardioembolic stroke. Stroke 2001:2762-2767.

17. Fagan SC, Nagaraja TN, Fenstermacher JD, Zheng J, Johnson M, Knight RA: Hemorrhagic transformation is related to the duration of occlusion and treatment with tissue plasminogen activator in a nonembolic stroke model. Neurol Res 2003, 25(4):377-382.

18. Fagan SC, Garcia JH: Hemorrhagic transformation in focal cerebral ischemia: influence of time to artery reopening and tissue plasminogen activator. Pharmacotherapy 1999 , I9(2): 139-142.

19. Chopp M, Li Y, Jiang N, Zhang RL, Prostak J: Antibodies against adhesion molecules reduce apoptosis after transient middle cerebral artery occlusion in rat brain. J Cereb Blood Flow Metab 1996:578-584.

20. Maier CM, Hsieh L, Yu F, Bracci P, Chan PH: Matrix metalloproteinase-9 and myeloperoxidase expression: quantitative analysis by antigen immunohistochemistry in a model of transient focal cerebral ischemia. Stroke 2004, 35(5): I 169-1 I 74.
21. Fagan SC, Hess DC, Hohnadel EJ, Pollock DM, Ergul A: Targets for vascular protection after acute ischemic stroke. Stroke 2004, 35(9):2220-2225.

22. Sir O, Fazal N, Choudry MA, Goris RJA, Gamelli RL, Sayeed MM Role of neutrophils in burn-induced microvascular injury in the intestine. Shock 2000: I I3-II7.

23. Knight RA, Barker PB, Fagan SC, Li Y, Jacobs MA, Welch KMA: Prediction of impending hemorrhagic transformation in ischemic stroke using magnetic resonance imaging in rats. Stroke 1998, 29:144-151.

24. Bederson JB, Pitts LH, Tsuji M, Nishimura MC, Davis RL, Bratowski J: Stroke 1986:472-476.

25. Terai K, Suzuki M, Miyata K: Amount of bleeding and hematoma size in the collagenase-induced intracerebral hemorrhage rat model. Neurochemical Research 2003, 28:779-785.

26. Lin TN, He YY, Wu G, Khan M, Hsu CY: Effects of brain edema on infarct volume in a focal cerebral ischemia model in rats. Stroke 1993, 24: |17-121.

27. Yang Y, Li Q, Miuyashita H, Howlett W, Siddiqui M, Shauaib A: Usefulness of postischemic thrombolysis with or without neuroprotection in a focal embolic model of cerebral ischemia. J Neurosurg 2000, 92:84I-847

28. Swanson RA, Morton MT, Tsao-Wu G, Savalos RA, Davidson C Sharp FR: A semiautomated method for measuring brain infarct volume. J Cereb Blood Flow Metab 1990, 1 0:290-293.

29. Hilali H, Simpkins A, Hill W, Waller J, Knight R, Fagan S: Single slice method for quantification of hemorrhagic transformation using direct ELISA. Neurol Research 2004:93-98.

30. Ergul A, Portik-Dobos V, Giulumian AD, Molero MM, Fuchs LC: Stress upregulates arterial matrix metalloproteinase expression and activity via endothelin $A$ receptor activation. Am J Physiol Heart Circ Physiol 2003, 285(5):H2225-32.
Publish with Biomed Central and every scientist can read your work free of charge

"BioMed Central will be the most significant development for disseminating the results of biomedical research in our lifetime. "

Sir Paul Nurse, Cancer Research UK

Your research papers will be:

- available free of charge to the entire biomedical community

- peer reviewed and published immediately upon acceptance

- cited in PubMed and archived on PubMed Central

- yours - you keep the copyright
BioMedcentral 